\title{
Paralisia obstétrica: Avaliação da técnica Sever-L'Episcopo modificada por Hoffer*
}

\section{Obstetric Paralysis: Evaluation of the Sever-L'Episcopo Technique Modified by Hoffer}

\author{
Antonio L. Severo ${ }^{10}$ Pedro G. L. Carvalho ${ }^{10}$ Marcelo B. Lemos ${ }^{10}$ Marcos C. Nunes ${ }^{10}$ \\ Marjurie Scaranto ${ }^{10}$ Fernando K. Barros ${ }^{2(1)}$
}

${ }^{1}$ Departamento do Instituto de Ortopedia e Traumatologia, Universidade Federal da Fronteira Sul, Campus do Hospital São Vicente de Paulo, Passo Fundo, RS, Brasil

2 Hospital Militar da Brigada Militar de Santa Maria, Santa Maria, RS, Brasil

\begin{abstract}
Endereço para correspondência Antônio Lourenço Severo, PhD, Departamento do Instituto de Ortopedia e Traumatologia, Universidade Federal da Fronteira Sul, Campus do Hospital São Vicente de Paulo, Rua Uruguai, 2050, Passo Fundo, RS, 99010-112, Brasil (e-mail: antoniolsevero@gmail.com; pesquisa2@hsvp.com.br).
\end{abstract}

Rev Bras Ortop 2020;55(6):787-795.

\section{Resumo \\ Palavras-chave \\ - ombro \\ - paralisia obstétrica \\ - contratura}

\section{Abstract}

Avaliar os resultados de uma série de 28 casos de paralisia obstétrica alta tratadas com a técnica Sever-L'Episcopo modificada por Hoffer, entre 2003 e 2016. As crianças (idade média, quatro anos e sete meses) com contratura em adução e rotação interna do ombro sem deformidades ósseas secundárias (Mallet classe II) foram submetidas ao alongamento do músculo peitoral e tenotomia do músculo subescapular associada à transferência do latissimus dorsi e músculo redondo maior para o músculo infraespinhal, movendo-se para a função de rotadores externos e elevadores. O seguimento médio foi de 3 anos e 10 meses. Ao final do estudo, 24 pacientes obtiveram excelentes escores de avaliação funcional, principalmente de abdução e rotação externa, passando de Mallet classe II para classe IV. Quatro pacientes ainda demonstraram algum grau de limitação de movimento global, passando da classe II para a classe III. Independentemente do ganho funcional final, todos os pacientes foram capazes de realizar tarefas que antes eram difíceis. Os dados deste estudo sugerem que a cirurgia de Hoffer é um método eficaz no tratamento das sequelas de paralisia obstétrica alta sem deformidades ósseas secundárias.

Evaluate the results of a series of 28 cases of high obstetric paralysis treated with the SeverL'Episcopo technique modified by Hoffer, between 2003 and 2016. Children (mean age, four years and seven months) with adduction contracture and internal rotation of the shoulder without secondary bone deformities (Mallet class II) underwent lengthening of the pectoralis major muscle and tenotomy of the subscapularis muscle associated with transfer of the latissimus dorsi and teres major muscle to the infraspinatus muscle, moving to the function of external rotators and elevators. The mean follow-up was three years and
Estudo realizado no Departamento do Instituto de Ortopedia e Traumatologia da Universidade Federal da Fronteira Sul, Campus do Hospital São Vicente de Paulo, Passo Fundo, RS, Brasil. recebido

14 de Agosto de 2019 aceito

10 de Março de 2020
DOI https://doi.org/

10.1055/s-0040-1712990. ISSN 0102-3616.
Copyright $\odot 2020$ by Sociedade Brasileira License terms de Ortopedia e Traumatologia. Published by Thieme Revinter Publicações Ltda, Rio de Janeiro, Brazil 
Keywords

- shoulder

- paralysis obstetric

- contracture
10 months. At the end of the study, 24 patients achieved excellent functional assessment scores, mainly of the abduction and external rotation, passing from Mallet class II to class IV. Four patients still demonstrated some degree of global movement limitation, passing from class II to class III. Regardless of the final functional gain, all patients were able to perform tasks that were previously difficult. The data from this study suggest that Hoffer's surgery is an effective method in the treatment of the sequelae of high obstetric paralysis without secondary bone deformities.

\section{Introdução}

A paralisia obstétrica (PO) do plexo braquial é uma desordem traumática causada pelo alongamento forçado de um ou mais componentes do plexo no parto. A incidência dessa condição nos países industrializados é de 0,5 a 3 por 1.000 nascidos vivos. ${ }^{1}$ Os fatores de risco obstétricos para lesão do plexo são alto peso ao nascer, trabalho de parto prolongado, apresentação pélvica, e distocia do ombro. ${ }^{2}$ Embora incomum, o plexo também pode ser ferido durante a cesariana ou apresentação cefálica.

A PO pode ser classificada de acordo com a gravidade, seguindo a classificação de Sunderland (graus I-V) de acordo com as raízes nervosas lesadas do plexo. ${ }^{2-4}$ Quanto ao grau de lesão, a PO pode ser agrupada em (1) paralisia maior ou paralisia de Erb-Duchenne com envolvimento das raízes $\mathrm{C5}$ e C6 e possível envolvimento da raiz C7; (2) A paralisia de Klumpke afeta as raízes de $\mathrm{C} 8$ a T1; e (3) a paralisia de todo o braço, com envolvimento de todas as raízes do plexo. ${ }^{2,3} \mathrm{~A}$ paralisia de Erb-Duchenne é o tipo mais comum, representando cerca de $75 \%$ dos casos. ${ }^{3,5}$

O exame físico de um recém-nascido com a variante ErbDuchenne mostra o membro superior afetado sem movimento ao lado do corpo e o cotovelo em extensão. $O$ reflexo de Moro está ausente deste lado. Edema e equimose na região supraclavicular podem estar associadas à fratura clavicular ou umeral. Os músculos deltoides e rotadores externos são comumente paralisados, enquanto o movimento dos dedos e punhos é mantido. ${ }^{2}$

A contratura em rotação medial associada à adução do ombro é a deformidade mais comum em crianças com plexopatia crônica. ${ }^{2}$ Com o tempo, por volta dos 2 anos de idade, a criança desenvolve subluxação/luxação posterior da articulação glenoumeral e uma deformidade articular glenoumeral. $^{6}$

Vários procedimentos cirúrgicos têm sido descritos na literatura para a correção dessa deformidade e suas limitações. Fairbank ${ }^{7}$ recomendou a seção da porção superior dos músculos peitoral e subescapular e a cápsula anterior do ombro. A principal complicação desta técnica é a luxação anterior tardia. ${ }^{2,7}$ Sever, ${ }^{8}$ por sua vez, recomendou a liberação do músculo subescapular sem capsulotomia prévia, prevenindo essa complicação. $^{2,3,8}$ L'Episcopo $^{9}$ associou a transferência do músculo redondo maior para uma posição lateral, transformando-o em um músculo rotador externo; ${ }^{2,3,9}$ Zachary $^{10}$ transferiu os latissimus dorsi e redondo maior como músculos rotadores externos. ${ }^{2,3,10}$ Zancolli $^{11}$ recomendou que apenas o músculo latissimus dorsi seja transferido após tenotomia e alongamento em "Z" come a tira do tendão distal passando atrás do úmero através do espaço quadrilateral e sendo suturada na tira tendinosa proximal com o ombro a $90^{\circ}$ de abdução. Além disso, Zancolli ${ }^{11}$ seccionou os músculos peitoral maior, subescapular e coracobraquial e a cabeça curta do bíceps. Ele também recomendou a transferência do peitoral maior para o tendão subescapular para manter a rotação medial. ${ }^{2,3,10}$ Hoffer et al. ${ }^{12}$ recomendaram transferência dos músculos redondo maior e latissimus dorsi para o manguito rotador. ${ }^{2,3,11}$ Pichon e Carlioz ${ }^{13}$ defenderam a ressecção da origem muscular subescapular. ${ }^{2,3}$ Cirurgias de tecido mole são indicadas em casos iniciais de contratura em adução e rotação medial que não melhoraram com órteses e alongamentos e que não apresentam deformidades ósseas. ${ }^{2,3,12}$

Miyazaki et al. ${ }^{14}$ sugeriram liberação articular anterior artroscópica associada à transferência de peitoral maior com enxerto de tendão homólogo alongado e reforçado (Aquiles ou tendão patelar) para a porção posterosuperior do tubérculo maior. Este procedimento foi indicado para aqueles com déficit funcional de rotação lateral do ombro, articulação congruente e sem deformidades glenoidais ou umerais, apresentando aumento na rotação lateral ativa e passiva, mas apresentou piora da rotação medial. Outros movimentos como elevação, mão-a-boca e mão-a-pescoço tiveram evolução menos consistente.

A principal cirurgia óssea é a osteotomia de desrotação do úmero, que posiciona o segmento distal na rotação lateral em casos de luxação posterior da articulação glenoumeral. ${ }^{2,3,12}$

Este estudo teve como objetivo e subjetivamente avaliar o resultado do tratamento e as sequelas da alta PO a técnica Sever-L'Episcopo modificada por Hoffer.

\section{Métodos}

Trata-se de um estudo retrospectivo de coorte longitudinal. $O$ escore de Mallet (-Fig. 1) foi utilizado para as avaliações objetivas pré e pós-cirúrgicas, enquanto o Questionário de Vida Diária foi utilizado para a avaliação subjetiva.

Entre janeiro de 2003 e outubro de 2016, 28 pacientes com sequelas de paralisía obstétrica foram submetidos a cirurgia no Serviço de Cirurgia e Microcirurgia de Membros Superiores do Instituto de Ortopedia e Traumatologia. O período de seguimento pós-operatório variou de 1 ano e 10 meses a 10 anos e 10 meses, representando uma média de 3 anos e 10 meses (-Tabela $\mathbf{1}$ ). 


\begin{tabular}{|c|c|c|c|c|c|c|}
\hline \multicolumn{7}{|c|}{$\begin{array}{l}\text { Classificação de Mallet modificada } \\
\text { (grau I = sem função, grau } \mathrm{V}=\text { função normal) }\end{array}$} \\
\hline & & Grau I & Grau II & Grau III & Grau IV & Grau V \\
\hline Abdução global & $\begin{array}{c}\text { Sem } \\
\text { possibilidade } \\
\text { de teste }\end{array}$ & Sem função & $230^{\circ}$ & $30^{\circ}$ a $90^{\circ}$ & $>90^{\circ}$ & Normal \\
\hline $\begin{array}{c}\text { Rotação externa } \\
\text { global }\end{array}$ & $\begin{array}{c}\text { Sem } \\
\text { possibilidade } \\
\text { de teste }\end{array}$ & Sem função & $\theta$ & $0^{\circ}$ & $>20^{\circ}$ & Normal \\
\hline Mão no pescoço & $\begin{array}{c}\text { Sem } \\
\text { possibilidade } \\
\text { de teste }\end{array}$ & Sem funçăo & Sem possibilidade & Dificil & Fácil & Normal \\
\hline Mão na coluna & $\begin{array}{c}\text { Sem } \\
\text { possibilidade } \\
\text { de teste }\end{array}$ & Sem funçăo & Sem possibilidade & S1 & T12 & Normal \\
\hline Mão na boca & \begin{tabular}{|c|} 
Sem \\
possibilidade \\
de teste
\end{tabular} & Sem funçăo & $\begin{array}{l}\text { Sinal de trompete } \\
\text { marcado }\end{array}$ & $\begin{array}{c}\text { Sinal de trompete } \\
\text { parcial }\end{array}$ & Abdução $<40^{\circ}$ & Normal \\
\hline Rotação interna & $\begin{array}{c}\text { Sem } \\
\text { possibilidade } \\
\text { de teste }\end{array}$ & Sem funçāo & Impossivel tocar & $\begin{array}{c}\text { Toca com flexão } \\
\text { de punho }\end{array}$ & $\begin{array}{l}\text { Palma da mão t } \\
\text { na barriga, sem } \\
\text { flexão de punho }\end{array}$ & \\
\hline
\end{tabular}

Fig. 1 Classificação de Mallet modificada (Adaptada da cirurgia da mão operativa de Green, 2017).

Participaram do estudo 17 pacientes do sexo masculino $(60,7 \%)$ e 11 pacientes do sexo feminino (39,3\%). Destes, 13 $(46,4 \%)$ tiveram envolvimento de lado esquerdo e 15 (53,6\%) do lado direito, com idades que variam de um ano a 14 anos e 8 meses (média de 4 anos e 7 meses).

Todos os pacientes atenderam aos critérios de inclusão de paralisia Erb-Duchenne com contratura rotacional interna e adução. A abdução do membro afetado foi $<30^{\circ}$, enquanto a rotação externa foi de $0^{\circ}$. Os pacientes não foram capazes de realizar tarefas com o membro afetado, como trazer a mão para a nuca ou para trás. Quando solicitados a levar a mão para a boca, mostraram dificuldade, simultaneamente abduzindo o ombro, flexionando o cotovelo e acentuando a lordose lombar (sinal do corneteiro), cumprindo os critérios do grau II da pontuação de Mallet - (Fig. 1).

Foram excluídos do estudo pacientes com paralisia total e baixa, com paralisia alta e luxação glenoumeral após o exame radiográfico, e aqueles que foram submetidos à cirurgia neurológica.
Todos os pacientes foram tratados cirurgicamente usando a técnica Sever-L'Episcopo modificada por Hoffer.

\section{Descrição da Técnica}

Um coxim é colocado no tórax superior para expor o membro superior (parte anterior e posterior) e a metade lateral do tórax. A incisão anterior é estendida do processo coracoide até o sulco deltopeitoral - (Fig. 2A). A veia cefálica é protegida e retraída junto com o músculo deltoide. $O$ intervalo entre os músculos deltoide e peitoral é aberto - (Fig. 2B). A cabeça curta do bíceps e do músculo coracobraquial são visualizadas e separadas do processo coracoide e afastadas para a visualização dos músculos redondo maior e latissimus dorsi -(Fig. 2C). Distalmente, no campo cirúrgico, o peitoral maior é alongado em Z; a metade distal de seu tendão é imediatamente desinserida da diafise umeral. Os cotos são suturados e o alongamento é alcançado (- Fig. 2D).

As inserções dos músculos latissimus dorsi e redondo maior são identificadas, incisadas e reparadas (-Fig. 2E) 
Tabela 1 Dados de 28 pacientes submetidos à técnica Sever-L’Episcopo modificada por Hoffer

\begin{tabular}{|c|c|c|c|c|c|c|c|}
\hline \multirow[b]{2}{*}{ Caso } & \multirow[b]{2}{*}{ Sexo } & \multirow[b]{2}{*}{$\begin{array}{l}\text { Lado } \\
\text { afetado }\end{array}$} & \multicolumn{2}{|c|}{ Pré-operatório } & \multicolumn{3}{|l|}{ Pós-operatório } \\
\hline & & & $\begin{array}{l}\text { Idade na } \\
\text { cirurgia } \\
(a+m)\end{array}$ & $\begin{array}{l}\text { Avaliação } \\
\text { funcional } \\
\text { (Mallet) }\end{array}$ & $\begin{array}{l}\text { Comprimento de } \\
\text { seguimento } \\
(a+m)\end{array}$ & $\begin{array}{l}\text { Avaliação } \\
\text { funcional } \\
\text { (Mallet) }\end{array}$ & $\begin{array}{l}\text { Questionário } \\
\text { da Vida Diária }\end{array}$ \\
\hline 1 & $\mathrm{~F}$ & $\mathrm{~L}$ & $6+4$ & II & $1+10$ & IV & $S$ \\
\hline 2 & $M$ & $\mathrm{~L}$ & $1+4$ & II & $1+10$ & IV & $S$ \\
\hline 3 & $\mathrm{M}$ & $\mathrm{R}$ & $4+8$ & II & $2+3$ & IV & $\mathrm{S}$ \\
\hline 4 & $\mathrm{M}$ & $\mathrm{R}$ & $9+3$ & II & $2+6$ & IV & $S$ \\
\hline 5 & $M$ & $\mathrm{~L}$ & $3+7$ & II & $2+9$ & IV & $S$ \\
\hline 6 & $M$ & $\mathrm{~L}$ & $4+6$ & II & $2+6$ & IV & $\mathrm{S}$ \\
\hline 7 & $\mathrm{M}$ & $\mathrm{R}$ & $5+9$ & II & $2+2$ & IV & $\mathrm{S}$ \\
\hline 8 & $\mathrm{~F}$ & $\mathrm{~L}$ & $4+5$ & II & $3+6$ & IV & $\mathrm{S}$ \\
\hline 9 & $\mathrm{~F}$ & $R$ & $2+4$ & II & $2+4$ & IIII & $\mathrm{S}$ \\
\hline 10 & $\mathrm{~F}$ & $R$ & $6+8$ & II & $2+2$ & IV & $\mathrm{S}$ \\
\hline 11 & $\mathrm{M}$ & $\mathrm{R}$ & $9+11$ & II & $2+4$ & IV & $S$ \\
\hline 12 & $\mathrm{~F}$ & $\mathrm{R}$ & $2+10$ & II & $2+0$ & III & $\mathrm{S}$ \\
\hline 13 & $\mathrm{~F}$ & $\mathrm{~L}$ & $2+11$ & II & $2+2$ & IV & $\mathrm{S}$ \\
\hline 14 & $\mathrm{M}$ & $\mathrm{R}$ & $3+6$ & II & $2+4$ & IV & $\mathrm{s}$ \\
\hline 15 & $\mathrm{~F}$ & $\mathrm{~L}$ & $2+9$ & II & $3+2$ & IV & $\mathrm{S}$ \\
\hline 16 & $\mathrm{~F}$ & $\mathrm{R}$ & $14+8$ & II & $7+8$ & III & $\mathrm{S}$ \\
\hline 17 & $\mathrm{~F}$ & $\mathrm{R}$ & $9+3$ & II & $4+7$ & IV & $S$ \\
\hline 18 & $M$ & $\mathrm{~L}$ & $4+5$ & II & $4+7$ & IV & $\mathrm{S}$ \\
\hline 19 & $M$ & $R$ & $3+11$ & II & $1+10$ & IV & $\mathrm{S}$ \\
\hline 20 & $M$ & $\mathrm{~L}$ & $1+8$ & II & $3+8$ & IV & $S$ \\
\hline 21 & $\mathrm{M}$ & $R$ & $9+6$ & II & $5+3$ & IV & $S$ \\
\hline 22 & $\mathrm{M}$ & $\mathrm{L}$ & $2+6$ & II & $10+10$ & IV & $\mathrm{S}$ \\
\hline 23 & $M$ & $R$ & 1 & II & 10 & IV & $S$ \\
\hline 24 & $\mathrm{M}$ & $\mathrm{R}$ & $4+3$ & II & $4+9$ & IV & $S$ \\
\hline 25 & $M$ & $\mathrm{~L}$ & $1+9$ & II & $3+7$ & III & $S$ \\
\hline 26 & $M$ & $R$ & 1 & II & $8+8$ & IV & $\mathrm{S}$ \\
\hline 27 & $\mathrm{M}$ & $\mathrm{L}$ & $1+6$ & II & $3+8$ & IV & $S$ \\
\hline 28 & $\mathrm{M}$ & $\mathrm{L}$ & $3+3$ & II & $4+8$ & IV & $S$ \\
\hline
\end{tabular}

Abreviaturas: m, meses; S,satisfeito; a, anos.

Fonte: SAME/Serviço Ambulatorial de Cirurgia do Membro Superior e Microcirurgia, campus do Hospital São Vicente de Paulo da Universidade Federal da Fronteira Sul.

após o músculo subescapular ser exposto e dissecado na cabeça do úmero. O tendão subescapular é alongado horizontalmente por um corte oblíquo feito usando um bisturi para dividir o tendão em metades superficial e profunda (-Fig. 2F). Isso permite a abdução e a rotação externa do ombro para cuidadosa visualização da inserção dos músculos latissimus dorsi e redondo maior, uma vez que a cápsula não é aberta.

No segundo estágio cirúrgico, uma incisão posterior de 7 a $8 \mathrm{~cm}$ é feita no intervalo entre os músculos deltoide e tríceps. O músculo deltoide é retraído superiormente e a cabeça longa do tríceps é retraída posteriormente para expor os músculos latissimus dorsi e redondo maior. Deve-se tomar cuidado para não ferir os nervos radial e axilar, os vasos uméris circunflexos posteriores e a artéria braquial profunda (-Figs. 3A, 3B). Posteriormente, os cotos reparados dos músculos latissimus dorsi e redondo maior são identificados e passados lateralmente à cabeça longa do músculo tríceps. $O$ ombro é colocado em abdução e rotação lateral máxima, e os tendões dos músculos latissimus dorsi e redondo maior agora músculos posteriores - são suturados na inserção do músculo infraespinhal e atingem o músculo supraespinhal, atuando como rotadores externos e elevadores em vez de rotadores internos (-Figs. 3C, 3D).

O membro operado é mantido em uma órtese com o braço na posição chamada "Estátua da Liberdade" durante 24 horas por dia durante o $1^{\circ}$ mês. Após esse período, no $2^{\circ}$ mês, o dispositivo é utilizado 2 horas pela manhã, 4 horas à tarde e 

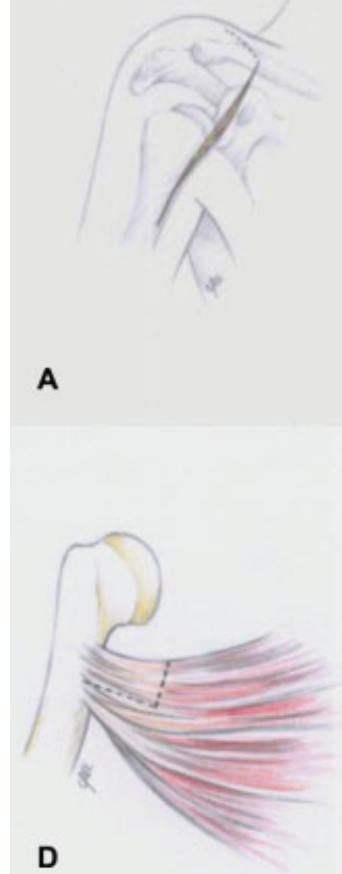

A
B

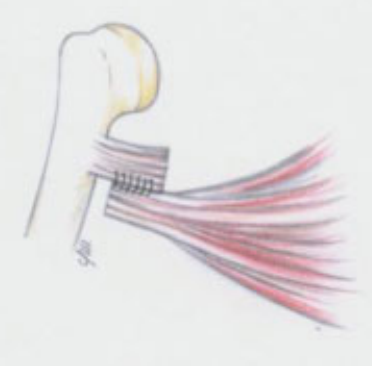

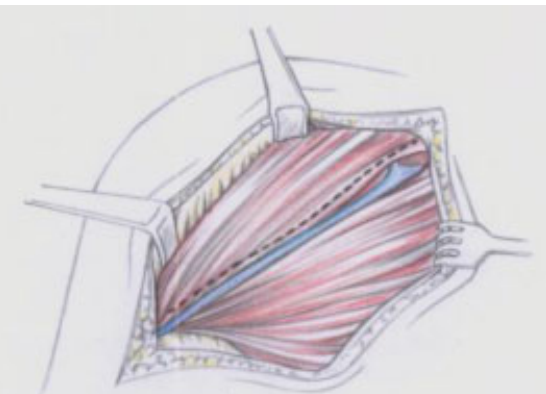

$\lambda$

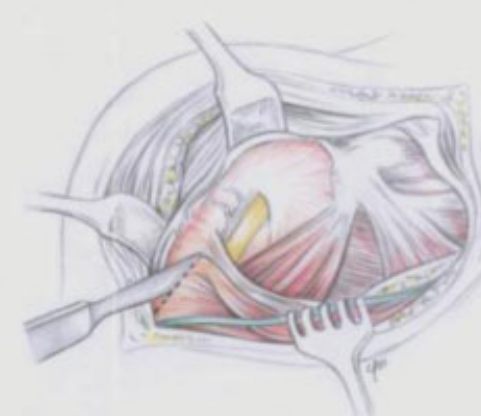

C

Fig. 2 (A) Incisão deltopeitoral anterior; (B) Veia cefálica no centro do campo e o intervalo aberto entre os músculos deltoide e peitoral (linha tracejada); (C) Retração muscular deltoide e exposição da inserção do músculo peitoral maior (dividido com bisturi); (D) alongamento em "Z" do músculo peitoral maior (seção em linhas tracejadas) e sutura dos cotos; (E) O tendão subescapular (linha tracejada mais superior) é dividido e alongado; os músculos latissimus dorsi e redondo maior são divididos e os cotos são reparados (linhas tracejadas inferiores); (F) Localização da divisão do tendão subescapular (alongado horizontalmente através de um corte oblíquo).

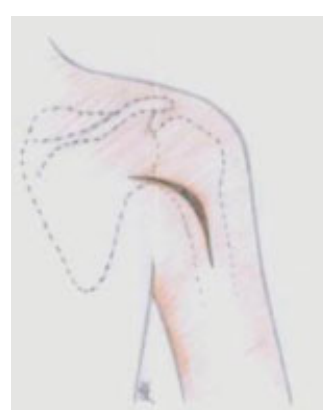

A
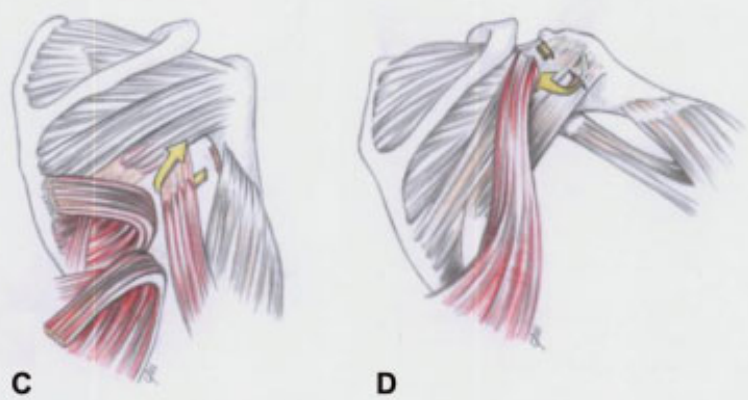

D

Fig. 3 (A) Incisão posterior no intervalo entre os músculos deltoide e tríceps. (B) Visualização posterior das inserções dos músculos latissimus dorsi e redondo maior e localização da área, incluindo estes tendões; (C) Os músculos latissimus dorsi e redondo maior são separados e passados posteriormente para a cabeça longa do músculo tríceps; (D) Com o ombro abduzido, os cotos são suturados no manguito rotador.

toda a noite, juntamente com exercícios fisioterapêuticos para ajudar o paciente a ganhar movimento. A partir do $3^{\circ}$ mês, a órtese é usada apenas à noite; seu uso pode ser prorrogado até o sexto mês pós-operatório, dependendo do desenvolvimento motor da criança = (Fig. 4).

Todos os pacientes (idade mínima, 1 ano e 10 meses; idade máxima, 10 anos e 10 meses) foram subjetivamente reavaliados no pós-operatório por meio de questionário respondido por seus responsáveis e reavaliados objetivamente por exame físico.

O Questionário da Vida Diária continha perguntas relacionadas ao grau de satisfação dos pais e à capacidade da criança de realizar tarefas antes impossíveis, como vestir-se sozinha, escovar os cabelos e realizar atividades recreativas.
Na avaliação objetiva, foram avaliados os graus de abdução e rotações externas e internas ativas, bem como a capacidade de levar a mão para a boca e a nuca. Devido a grandes discrepâncias entre observadores, optou-se por classificar cada paciente de acordo com sua amplitude de movimento expressa como pontuação de Mallet ${ }^{15}$ - (Fig. 1).

\section{Resultados}

Nossos pacientes foram acompanhados por uma média de 3 anos e 10 meses. Ao final do período de seguimento, 24 pacientes apresentaram excelentes ganhos de movimento, passando de escores de Mallet do grau II para o grau IV, com 


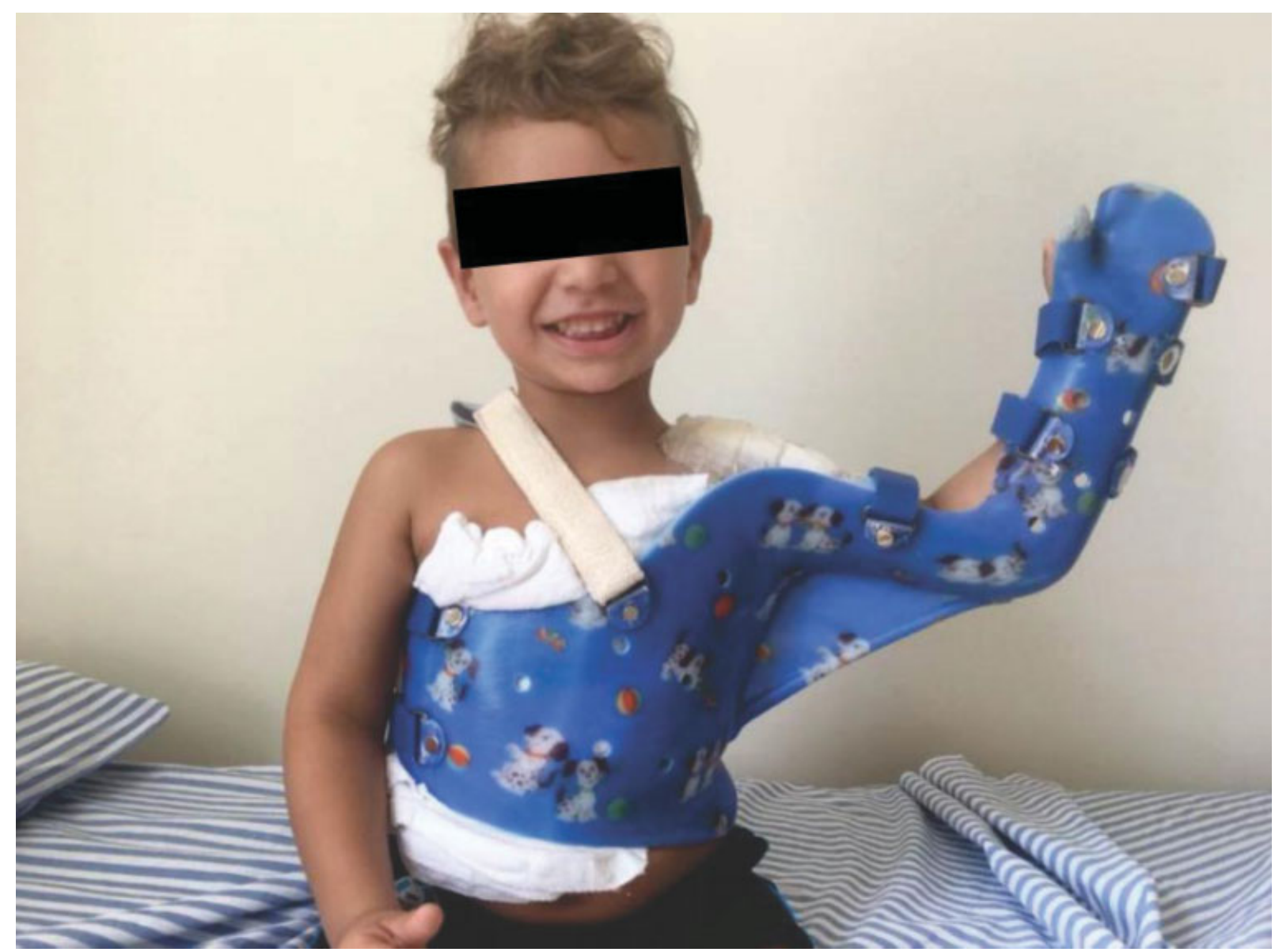

Fig. 4 Órtese usada para manter o paciente na posição "Estátua da Liberdade" no pós-operatório.

20 tendo tais resultados no $3^{\circ}$ mês pós-operatório quando a fisioterapia foi intensificada. Ao final do $6^{\circ}$ mês pós-operatório, 25 pacientes alcançaram o grau IV de Mallet. Embora o estado de um paciente tenha regredido ao grau III durante o seguimento, ainda era clinicamente melhor do que antes da cirurgia. Assim, ao final do seguimento, 4 pacientes (tempo de seguimento: 2 anos e 4 meses, 2 anos, 3 anos e 7 meses, e 7 anos e 8 meses) foram classificados como grau III. Portanto, ao final do seguimento, $85 \%$ foram classificados como grau IV e $15 \%$ classificados como grau III.

De todos os pacientes, quatro evoluíram com perda parcial da rotação interna do ombro, conseguindo durante o exame o toque da mão logo acima da região glútea, mesmo após o tratamento fisioterapêutico, evento um tanto esperado em alguns casos devido à transposição de rotadores internos para externos.

Independentemente dos resultados, todos os membros da família ficaram satisfeitos com os resultados e todas as crianças começaram a realizar tarefas que antes eram impossíveis para eles, como vestir-se sozinhas, escovar os cabelos e participar de esportes (-Figs. 5, 6).

\section{Discussão}

Os pacientes do estudo apresentaram idade média de 4 anos e 7 meses (mínimo, 1 ano; máximo, 14 anos e 8 meses). Esta é uma faixa etária em que as complicações raramente resultam de contratura muscular. O consenso na literatura é que a tenotomia e as cirurgias de transferência muscular são a primeira opção no tratamento das sequelas de PO em pacientes mais jovens que ainda não desenvolveram luxação e incongruência articular. As osteotomias são estritamente recomendadas para casos mais velhos, geralmente crianças de 10 a 14 anos de idade, em que a luxação crônica já levou a um comprometimento parcial da cabeça do úmero ou glenoide. ${ }^{2-5,8,11}$

Giostri et al. ${ }^{16}$ compararam 20 pacientes. Treze de seus pacientes foram designados para o Grupo A, tiveram idade média de 6 anos e 3 meses, e foram submetidos a cirurgia utilizando a técnica L'Episcopo modificada por Tachdjian. Destes, cinco foram classificados como grau II, seis como grau III e dois como grau IV na classificação de Mallet. Os outros 7 foram designados para o Grupo B, tiveram idade média de 7 anos e 11 meses, e foram submetidos à osteotomia do úmero. Destes, cinco foram classificados como grau IV e dois classificados como grau III. Os autores concluíram que os resultados da osteotomia foram estatisticamente superiores aos da transferência tendinosa. ${ }^{16}$ Em contrapartida, o presente estudo apresentou resultados satisfatórios com 24 (85\%) pacientes classificados como grau IV e 4 (15\%) classificados como grau III apenas com transferência muscular, excluindo casos de osteotomia. Sugerimos que os resultados favoráveis podem ser explicados pela menor idade média de 


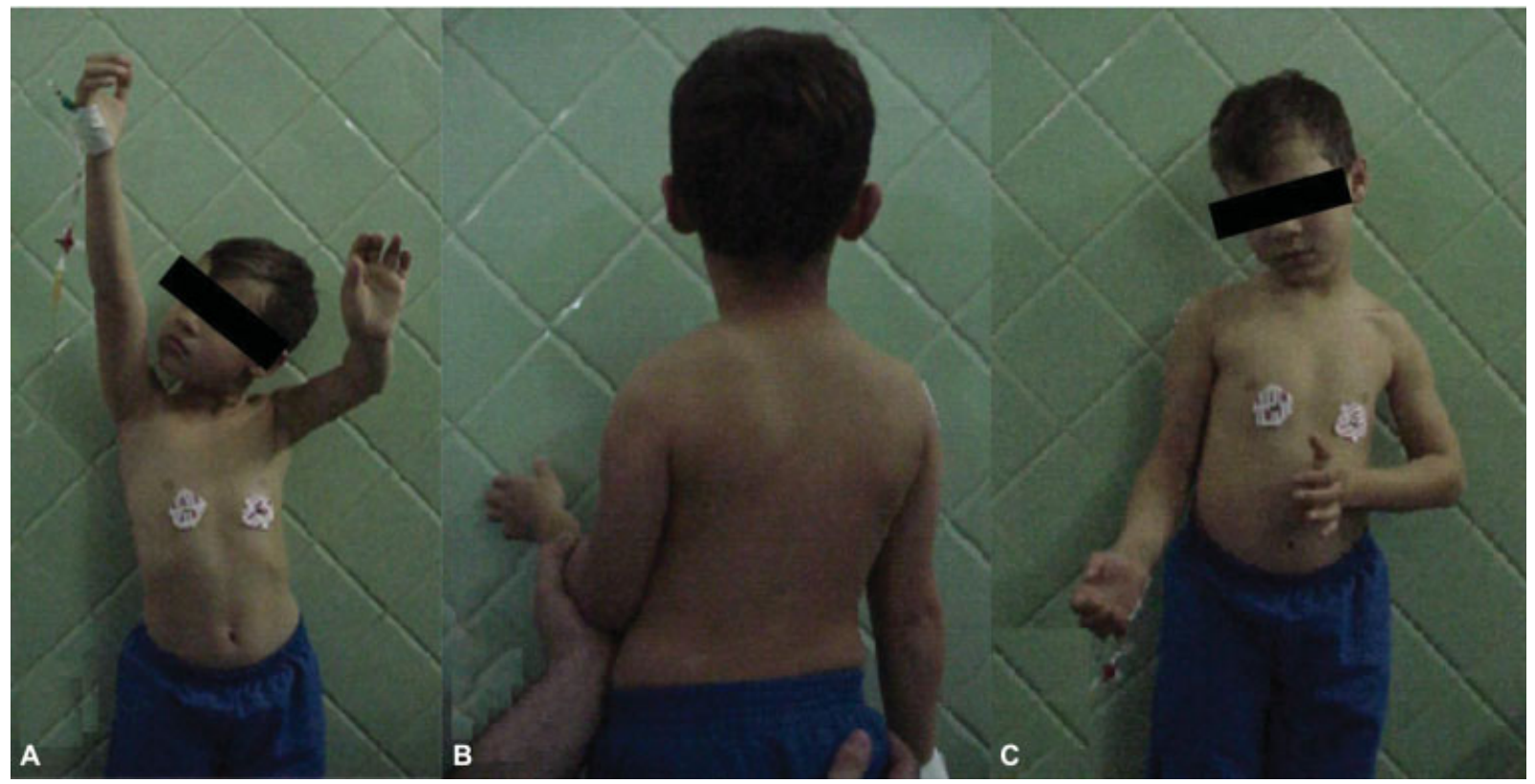

Fig. 5 (A, B e C) I.D.S., 5 anos, pré-operatório, com sequelas de paralisia obstétrica, bem como limitações na elevação/abdução e rotações externas e internas (Mallet grau II).

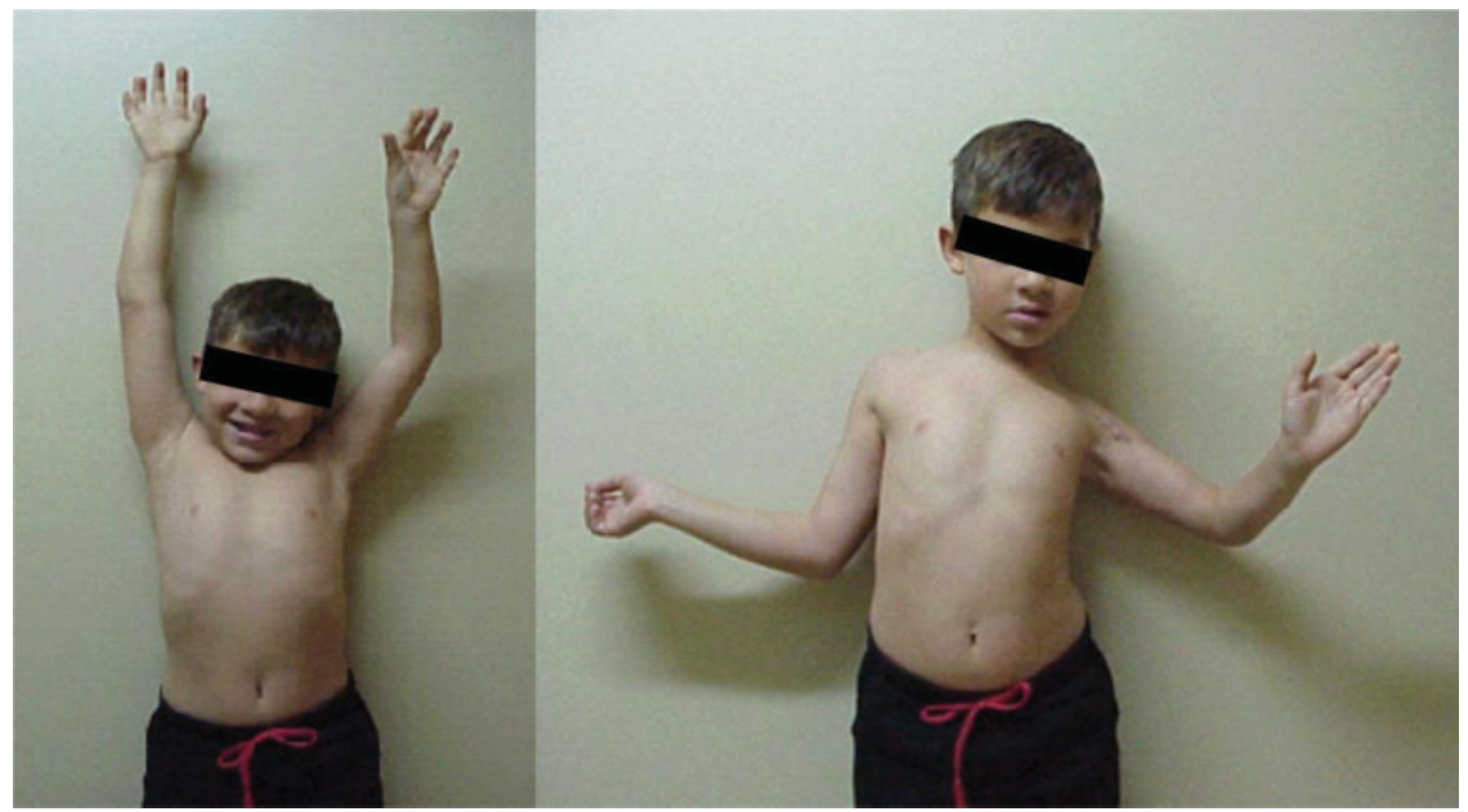

Fig. 6 Mesmo paciente apresentado na - Fig. 5 aos quatro meses de pós-operatório. Elevações superiores a $90^{\circ}$ e rotação externa superior a $20^{\circ}$ são evidentes (Mallet grau IV).

4 anos e 7 meses e o uso de uma técnica que eleve os músculos latissimus dorsi e redondo maior para o músculo infraespinhal.

Lopes et al. ${ }^{17}$ avaliaram os resultados obtidos em 7 pacientes com idade média de 14 anos e 10 meses que apresentaram cabeça umeral subluxada e/ou deformada e foram submetidos à osteotomia de rotação externa do úmero. Os autores consideraram os bons resultados como aqueles que consistem na realização da rotação externa ativa $>20^{\circ}$ e na capacidade de trazer a mão para a boca e para a cabeça. ${ }^{17}$ Observaram melhora com cirurgia em cinco pacientes. Embora este estudo tenha excluído todos os casos de luxação posterior da cabeça do úmero ou deformidades glenohumeral, há concordância na literatura de que a osteotomia é recomendada para esses casos.

Vieira et al. ${ }^{6}$ por sua vez, avaliaram 16 pacientes com idade entre 1 ano e 6 meses e 5 anos e 10 meses com sequelas de PO, contratura em adução, rotação interna e deslocamento posterior do ombro que foram submetidos a procedimento cirúrgico de centralização da cabeça umeral por osteotomia de desrotação interna possivelmente associada a procedimentos de tecido mole (cirurgia utilizando a técnica Sever-L'Episcopo). Os autores concluíram que a técnica deve ser utilizada em 
pacientes sem deformidades ósseas importantes em que a redução articular com congruência seja possível e realizada entre 2 e 8 anos de idade devido ao grande potencial de remodelação óssea. ${ }^{6}$ Nos casos de osteotomias para deformidade glenoumeral com ou sem luxação posterior da cabeça umeral identificada em tomografia computadorizada ou ressonância magnética, é importante ressaltar a importância do uso da classificação de Waters. ${ }^{4}$ Esta classificação consiste em sete tipos: tipo I, articulação glenoumeral normal; Tipo II, hipoplasia mínima da cavidade glenoide (aumento da retroversão $>5^{\circ}$ ); Tipo III, subluxação posterior da cabeça umeral; Tipo IV, desenvolvimento de uma cavidade glenoide falsa; Tipo V, retroversão posterior da cabeça umeral; Tipo VI, luxação posterior da cabeça umeral; e tipo VII, interrupção do crescimento da cabeça umeral. Com base nesta classificação, concordamos com Viera et al. ${ }^{6}$ que quando há o potencial de remodelação óssea, a osteotomia derrotatória interna é recomendada para os tipos I a III, enquanto a osteotomia derrotatório externa, como descrito por Giostri et al. ${ }^{16}$ e Lopes et al., ${ }^{17}$ é recomendado para os tipos IV a VII.

Pichon e Carlioz ${ }^{13}$ e Lopes Filho ${ }^{3}$ sugeriram que a liberação das estruturas contraturadas e a transferência do tendão sejam realizadas em diferentes cirurgias devido à necessidade de mobilização precoce após a liberação e que o paciente permaneça imobilizado após a transferência. ${ }^{3}$ Gilbert et al. ${ }^{18}$ recomenda-se a desinserção do subescapular na primeira cirurgia porque a maioria de seus pacientes com menos de dois anos de idade se beneficiou do uso deste procedimento apenas com riscos mínimos de recidiva da contratura. Para esses autores, a idade foi um fator importante na indicação da associação da transferência tendinosa. ${ }^{18}$ Os resultados do presente estudo concordam que a idade precoce é um fator determinante para a realização e o sucesso da liberação e transferência do tendão. Entretanto, no presente estudo, o tendão foi liberado na primeira cirurgia de acordo com a preferência cirúrgica dos autores.

Este estudo concorda com Chung e Nissenbaum ${ }^{19}$ e Narakas, ${ }^{20}$ que recomendam que a transferência do tendão ocorra ao mesmo tempo que a liberação da estrutura com contratura. Sugerimos que os dois procedimentos realizados em conjunto promovam a restauração do equilíbrio muscular e previnam a recorrência da deformidade. Neste estudo, o alongamento em " $Z$ " do músculo peitoral maior, além da tenotomia do músculo subescapular requer imobilização pós-operatória, independentemente da transferência do tendão.

A transferência dos músculos latissimus dorsi e redondo maior para a porção posterior do tubérculo maior, onde o músculo infraespinhal é inserido, potencializa a rotação externa ativa. ${ }^{21,22}$ Comtet et al., ${ }^{21}$ analisando a biomecânica da transferência do tendão, concluíram que os músculos inseridos nas tuberosidades são mais eficientes do que aqueles inseridos na diáfise umeral, como afirma Zachary. ${ }^{10}$ O ganho de abdução é o resultado de uma ação deltoide de forma mais eficiente devido ao efeito estabilizador do manguito rotador na junta glenoumeral reforçada pela transferência do latissimus dorsi. Phipps e Hoffer $^{22}$ chamaram o fenômeno da estabilização dinâmica da cabeça do úmero de "force couple".
Hoffer, $^{12}$ no artigo original, apresentou 11 pacientes com sequelas de $\mathrm{PO}$ que foram submetidos à cirurgia utilizando a técnica modificada de Sever-L'Episcopo. O seguimento variou de 2 anos a 6 anos e 8 meses. Todas as 11 crianças apresentaram melhor rotação externa e abdução. Nove pacientes passaram do grau II para o grau IV. Dois casos apresentaram rotação externa $<20^{\circ}$, passando do grau II para o grau III. Em um dos casos, o autor atribuiu o resultado ruim à falha na liberação do músculo subescapular. Todos os pacientes tiveram abdução $>90^{\circ}$ (grau IV). ${ }^{12}$ O presente estudo reproduziu os resultados de Hoffer et al. ${ }^{12}$ com um período de acompanhamento que variou de janeiro de 2003 a agosto de 2018. Vinte pacientes tiveram um ganho importante na abdução e rotações externa e interna no terceiro mês pós-operatório, sendo classificados como Mallet IV, enquanto quatro pacientes ainda apresentavam certa limitação no movimento de rotação interna, permanecendo na classe III. A fisioterapia foi então intensificada para ganho funcional; ao final do $6^{\circ}$ mês pós-operatório, esses pacientes passaram do grau III para a classificação grau IV. Assim, 24 de 28 (85\%) pacientes passaram do grau II para o grau IV após a liberação e transferência muscular.

Terzis e Kokkalis ${ }^{23}$ realizaram um estudo após avaliar 68 casos com diferentes cirurgias (tecidos moles e osteotomias) para o tratamento de paralisia obstétrica. Como resultado de cirurgias com transferência de latissimus dorsi com ou sem a liberação de redondo maior, $\sim 28 \%$ dos pacientes tiveram dificuldade em realizar atividades de vestir na linha média do corpo, mas afirmaram que essa perda foi suplantada pela melhora da função do membro. Da mesma forma, Pearl et al. ${ }^{24}$ descrevem que a perda de rotação interna deve ser esperada ao realizar qualquer procedimento que promova o movimento na direção oposta. Em seu estudo, realizaram liberação artroscópica da cápsula anterior e tendão subescapular, com transferência do latissimus dorsi para o manguito rotador e manutenção de redondo maior no sítio anatômico. Obteve perda parcial da rotação interna, porém uma melhora da amplitude de movimento da rotação externa e função do membro, fator que representa ganho muito mais importante para a função global.

Cabral et al. ${ }^{25}$ realizaram um estudo prospectivo com 16 pacientes após a cirurgia modificada de Sever-L'Episcopo. Obtiveram bons resultados, com melhoria de pelo menos 1 estágio na escala de Mallet. Relataram que, embora tenha sido observada uma importante melhora na rotação externa, tevese a impressão de que os pacientes apresentavam alguma dificuldade em atividades que dependiam da rotação interna do ombro, como abotoamento de camisas. No entanto, apenas dois pacientes apresentaram restrição no posicionamento da mão nas costas, o que supôs a existência de possível adaptação ao longo do tempo.

Em um acompanhamento a longo prazo de 10 casos de PO tratados pela técnica Hoffer, Suenaga et al. ${ }^{26}$ relataram que dois pacientes não apresentaram ganhos funcionais finais satisfatórios; pelo contrário, permaneceram no grau II. O tempo médio pós-operatório neste estudo foi de 11 anos. 0 resultado ruim em um caso foi atribuído à atrofia grave do músculo latissimus dorsi; no outro caso, foi atribuído ao fato de que o músculo latissimus dorsi não foi transferido durante a cirurgia. No entanto, os autores consideram a técnica cirúrgica 
de Hoffer adequada para a reconstrução e manutenção a longo prazo da função ativa do ombro afetado. ${ }^{26}$

Vários autores concordam que, independentemente do ganho funcional do membro operado, a manutenção da cabeça umeral no glenoide previne deformidades articulares marcadas que levariam à instabilidade do ombro e à falta de função. ${ }^{2-5,8,11}$

\section{Conclusão}

A técnica cirúrgica de alongamento do músculo peitoral maior e tenotomia do músculo subescapular associada à transferência dos músculos latissimus dorsi e redondo maior para o manguito rotador, como descrito por Hoffer, é eficaz para o tratamento de sequelas de Erb-Duchenne em PO. O procedimento é recomendado para todas as crianças com PO alta, contratura em adução e rotação interna sem deformidades ósseas secundárias. A técnica visa alcançar ganho funcional, principalmente de abdução e rotação externa, e permite que a criança realize atividades antes impossíveis.

Conflito de Interesses

Os autores não têm conflito de interesses a declarar.

\section{Referências}

1 de Luna Cabral JR, Crepaldi BE, Sambuy MT, da Costa AC, Abdouni YA, Chakkour I. Avaliação da função do membro superior nos pacientes com paralisia obstétrica após cirurgia de Sever-L'Episcopo modificada. Rev Bras Ortop 2010;47(04):451-454

2 Tachdjian MO. Distúrbios traumáticos: paralisia obstétrica do plexo braquial. In: Mihran O, Tachdjian MO, editores. Ortopedia pediátrica. $2^{\mathrm{a}}$ ed. São Paulo: Manole; 1995:2009-2020

3 Lopes Filho JD. Tratamento cirúrgico das seqüelas da paralisia obstétrica no ombro [dissertação]. São Paulo: Faculdade de Ciências Médicas da Santa Casa de São Paulo; 2001

4 Waters PM, Smith GR, Jaramillo D. Glenohumeral deformity secondary to brachial plexus birth palsy. J Bone Joint Surg Am 1998;80(05):668-677

5 Galbiatti JA, Faloppa F. Paralisia obstétrica. In: Hebert S, Xavier R, Pardini AG Jr, Barros Filho TEP. Ortopedia e traumatologia: princípios e prática. 3a. ed. Porto Alegre: Artmed; 2003:830-838

6 Vieira LA, Poderoso MA, Gonçalves MCK, et al. A osteotomia de centralização da cabeça umeral, na luxação posterior do ombro, sequela de paralisia obstétrica. Rev Bras Ortop 2004;39(11/12): 661-669

7 Fairbank HAT. A lecture on Birth Palsy: Subluxation of the shoulder-joint in infants and young children. Lancet 1913;181 (4679):1217-1223

8 Sever JW. Obstetrical paralysis. Surg Gynecol Obstet 1927;44:547-549
9 L'Episcopo JB. Tendon transplantation in obstetrical paralysis. Am Surg 1934;25:122-125

10 Zachary RB. Transplantation of teres major and latissimus dorsi for loss of external rotation at shoulder. Lancet 1947;2(6482):757

11 Zancolli EA. Classification and management of the shoulder in birth palsy. Orthop Clin North Am 1981;12(02):433-457

12 Hoffer MM, Wickenden R, Roper B. Brachial plexus birth palsies. Results of tendon transfers to the rotator cuff. J Bone Joint Surg Am 1978;60(05):691-695

13 Pichon F, Carlioz H. [Disinsertion of the subescapular muscle in the treatment of obstetric paralysis of the upper limb (author's transl)]. Chir Pediatr 1979;20(02):135-141

14 Miyazaki AN, Checchia CS, Checchia SL, Fregoneze M, Santos PD, Sella GV. Paralisia obstétrica: liberação artroscópica anterior do ombro e transferência do grande dorsal com enxerto homólogo. Rev Bras Ortop 2016;51(03):319-328

15 Mallet J. Paralysie obstétricale du plexus brachial. Traitment des sequelles. Rev Chir Orthop Repar Appar Mot 1972;58 (Suppl 1):166-168

16 Giostri GS, Machezini EJ, Pasin AP. Rotação interna na paralisia obstétrica: comparação dos resultados dos procedimentos de Sever-L'Episcopo e osteotomia derrotadora do úmero. Rev Bras Ortop 1996;31(01):33-35

17 Lopes EI, Chackkour I, Gomes MD, Cauchiolli CA, Ramirez JF, Lopes Filho JD. Osteotomia de rotação externa do úmero no tratamento das deformidades em rotação interna do ombro nas sequelas de paralisia obstétrica. Rev Bras Ortop 1996;31(04): 322-326

18 Gilbert A, Romana C, Ayatti R. Tendon transfers for shoulder paralysis in children. Hand Clin 1988;4(04):633-642

19 Chung SM, Nissenbaum MM. Obstetrical paralysis. Orthop Clin North Am 1975;6(02):393-400

20 Narakas AO. Muscle transpositions in the shoulder and upper arm for sequelae of brachial plexus palsy. Clin Neurol Neurosurg 1993; 95(Suppl):S89-S91

21 Comtet JJ, Herzberg G, Naasan IA. Biomechanical basis of transfers for shoulder paralysis. Hand Clin 1989;5(01):1-14

22 Phipps GJ, Hoffer MM. Latissimus dorsi and teres major transfer to rotator cuff for Erb's palsy. J Shoulder Elbow Surg 1995;4(02): 124-129

23 Terzis JK, Kokkalis ZT. Outcomes of secondary shoulder reconstruction in obstetrical brachial plexus palsy. Plast Reconstr Surg 2008;122(06):1812-1822

24 Pearl ML, Edgerton BW, Kazimiroff PA, Burchette RJ, Wong K. Arthroscopic release and latissimus dorsi transfer for shoulder internal rotation contractures and glenohumeral deformity secondary to brachial plexus birth palsy. J Bone Joint Surg Am 2006;88(03):564-574

25 de Luna Cabrai JR, Crepaldi BE, de Sambuy MT, da Costa AC, Abdouni YA, Chakkour I. Evaluation of upper-limb function in patients with obstetric palsy after modified Sever-L'Episcopo procedure. Rev Bras Ortop 2015;47(04):451-454

26 Suenaga N, Minami A, Kaneda K. Long-term results of multiple muscle transfer to reconstruct shoulder function in patients with birth palsy: eleven-year follow-up. J Pediatr Orthop 1999;19(05): 669-671 